\title{
Niveles de Urbanización, Uso de Televisión y Video-juegos en Niños Colombianos: Posibles Implicaciones en Salud Pública
}

\section{Possible public health implications regarding associations between the degree of urbanisation and electronic media exposure amongst Colombian children}

\author{
Luis F. Gómez ${ }^{1}$, Diego I. Lucumí1,2, Diana C. Parra ${ }^{3}$ y Felipe Lobelo ${ }^{4}$
}

\begin{abstract}
1 División de Salud Fundación FES Social. Bogotá Colombia. Ifgomez@fundacionfes.org; dilucumi@fundacionfes.org

2 Escuela de Medicina. Universidad Pedagógica y Tecnológica de Colombia.

3 Prevention Research Center in St. Louis. George Warren Brown School of Social Work. Washington University in St. Louis. dparrape@gmail.com

4 Department of Exercise Science, Arnold School of Public Health, University of South Carolina, Columbia, SC. Centers for Disease Control and Prevention, Division of Nutrition, Physical Activity and Obesity, Physical Activity and Health Branch, CDC/WHO Collaborating Center for Physical Activity and Health. lobelo@mailbox.sc.edu
\end{abstract}

Recibido 30 Enero 2008/Enviado para Modificación 15 Agosto/Aceptado 29 Agosto 2008

\section{RESUMEN}

Objetivo Examinar la asociación entre los niveles de urbanización y el uso excesivo de televisión y videojuegos (2 horas o más) en niños colombianos de 5 a 12 años. Métodos Un análisis secundario fue realizado a partir de los datos provenientes de 13.090 niños entre 5 a 12 años de edad que fueron seleccionados en la Encuesta Nacional de la Situación Nutricional en Colombia 2005, a los cuales se les determinó el tiempo dedicado a ver televisión y jugar con video juegos, a través de la información proveniente de uno de los padres o acudientes. Cinco niveles de urbanización fueron determinados mediante una adaptación de las categorías definidas por el Departamento Nacional de Planeación. Un modelo de regresión logística fue llevado a cabo, el cual considero potenciales variables de confusión.

Resultados Se observó un incremento gradual de la probabilidad de ver dos o más horas televisión o video juegos a medida que el nivel de urbanización era mayor, la cual fue estadísticamente significativa a partir del nivel de urbanización 3 (nivel 2 OR de prevalencia: 1,33 IC 95 \%: 0,89-1,99; nivel 3: 1,35: 1,00-1,80; nivel 4: 1,61: 1,16-2,23 y nivel 5: 1,70: 1,17-2,46).

Conclusión Los hallazgos de este estudio muestran como el uso excesivo de televisión y video juegos en niños esta asociado a mayores niveles de urbanización. Debido a las implicaciones que tiene para la salud de los niños la exposición excesiva a la televisión y otras formas de entretenimiento electrónico, es importante monitorear este comportamiento y entender sus determinantes.

Palabras Clave: Urbanización, televisión, juegos de video, salud pública (fuente: DeCS, BIREME). 


\section{ABSTRACT}

Objective Determining the association between the degree of urbanisation and excessive television-viewing and video-game use amongst children aged 5 to 12 years old.

Methods Data from the Colombian National Nutritional Survey 2005 was used in the analysis. Television viewing and video-game use was determined through parental reports for 13,090 children and classified as being excessive (e" 2 hours/day) or suitable ( $<2$ hours/day). Five levels of urbanisation were determined using criteria from the National Planning Department and the population size of the rural or urban settings included in the survey. Multiple logistical regression analysis was conducted while taking potential confounders into account.

Results There was a gradual increase in the probability of TV or videogames being used for 2 hours or more as the degree of urbanisation increased. This association was statistically significant for urbanisation level 3 and higher (urbanisation level 2, OR=1,33: 0,89-1,99 $95 \% \mathrm{Cl}$; urbanisation level 3, 1,35=OR: 1,00-1,80 $95 \% \mathrm{Cl}$; urbanisation level 4, 1,61=OR: 1,16-2,23 $95 \% \mathrm{Cl}$ and urbanisation level 5, 1,7=OR: 1,17-2,46 $95 \% \mathrm{Cl})$.

Conclusion Colombian children living in areas of high-to-moderate urbanisation and population density are at a higher risk of excessive exposure to electronic media entertainment. Due to its multiple health implications (in particular obesity and cardiometabolic health), such sedentary behaviour should be monitored and its determinants in the Colombian paediatric population must be understand for making effective public health interventions.

Key Words: Urbanisation, television, video-games, public health (source: MeSH, NLM).

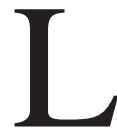

os vínculos entre hábitos televisivos, entretenimiento electrónico y salud en niños están enmarcados en un contexto de crecientes procesos de urbanización y globalización, que han estado acompañadas de intensas estrategias de la industria de mercado dirigidas a conocer preferencias del público infantil y a publicitar diferentes productos $(1,2)$. En este contexto, la población infantil no solo es considerada como grupo de consumo, sino además como un agente involucrado en la decisión del gasto familiar (3).

Son diversos los estudios que identifican los potenciales efectos positivos o negativos que tiene la televisión en la salud y desarrollo psicomotor de los niños (4-9). La televisión puede ser considerada como una herramienta efectiva de comunicación que puede ayudar a los niños a ampliar experiencias y conocimientos y, en algunos casos, puede favorecer procesos de aprendizaje. Algunos programas educativos diseñados para niños mayores de 3 años, han sido ampliamente evaluados, evidenciando un efecto significativo en el desarrollo del lenguaje, estimulando la imaginación y la fantasía (4). 
De igual manera, han sido identificados múltiples efectos negativos de los hábitos de televisión y el uso de video-juegos sobre la salud de los niños. Diversos estudios han encontrado que el uso excesivo de televisión y video juegos esta positivamente asociado con menores niveles de actividad física y una mayor posibilidad de padecer o desarrollar obesidad tanto en la niñez como en la adultez (5-9). En este sentido, los mensajes publicitarios transmitidos en televisión, han sido utilizados por la industria de alimentos como una estrategia efectiva para mercadear sus productos en la audiencia infantil (1). La mayoría de estos alimentos publicitados tienen una alta densidad energética, como bebidas gaseosas, comidas rápidas, dulces y pasteles; y es por el contrario inusual encontrar alguno de estos mensajes relacionados con el consumo de frutas y verduras (10-11). Los efectos de esta exposición publicitaria fueron estudiados por Dietz et al, quienes encontraron que el tiempo dedicado a ver televisión en Estados Unidos estaba positivamente correlacionado con la demanda, compra y consumo de los alimentos publicitados en este medio (12).

La televisión ha sido vinculada además, con trastornos del sueño, conductas agresivas, problemas de interacción social y disminución del tiempo dedicado a la lectura y al trabajo escolar (13-17).

En Colombia, la Comisión Nacional de Televisión, como autoridad encargada de regular la televisión pública y privada, ha definido un compromiso dirigido a mejorar la calidad de la televisión infantil y a controlar la exposición que pueda tener este público a ciertos productos que tengan impacto sobre la salud pública (18). Para lo anterior, esta entidad ha reconocido la necesidad de fomentar estudios sobre los aspectos que se relacionan con televisión e infancia (18).

Con el fin de diseñar e implementar estrategias de intervención que atenúen los efectos negativos de la exposición excesiva al entretenimiento electrónico (televisión y video-juegos) en la población colombina, se requiere profundizar acerca de los factores asociados a este hábito.

Los vínculos entre procesos de urbanización y hábitos televisivos y videojuegos no han sido suficientemente estudiados y entendidos en América Latina. Es así como este estudio busca determinar la asociación entre los niveles de urbanización y el uso excesivo de televisión y videojuegos en niños colombianos de 5 a 12 años. Dados los potenciales efectos de esta asociación sobre la salud de los niños, los resultados de este estudio podrán servir para soportar acciones tendientes a promover alternativas para el uso activo del tiempo libre, 
diseñar políticas públicas frente al contenido de la televisión y otras formas de entrenamiento electrónico y orientar investigaciones que permitan establecer el impacto real de estos hábitos ante crecientes problemas de salud, como la obesidad infantil en Colombia.

\section{MÉTODOS}

Tipo de estudio

Este estudio consistió en un análisis secundario de la información obtenida en la Encuesta Nacional de la Situación Nutricional 2008, la cual fue financiada por el Instituto Colombiano de Bienestar Familiar (ICBF) (18). Esta encuesta consistió en una medición transversal, llevada a cabo para determinar la prevalencia de problemas nutricionales y algunas condiciones de salud en la población colombiana. Para tales propósitos se diseñó una muestra probabilística, de conglomerados y multietápica, en la cual los municipios fueron considerados como las unidades primarias de muestreo.

Treinta y siete por ciento de la población entrevistada tenía una edad comprendida entre los 5 y 12 años, grupo a partir del cual se realizaron los análisis de este estudio.

Procesos de medición y recolección de la información

Una encuesta estructurada fue aplicada por profesionales nutricionistas a los padres o acudientes de los niños, con previo consentimiento oral. El grupo encuestador recibió una capacitación de 4 semanas sobre técnicas de aplicación de cuestionarios en encuestas poblacionales. La tasa de respuesta obtenida en el grupo de edad definido para este estudio fue del 94,3\%.

El estudio fue aprobado por el comité de ética de la Encuesta Nacional de la Situación Nutricional.

Variable resultado

Se definió como variable resultado el tiempo dedicado a ver televisión y jugar con video juegos, teniendo en cuenta la siguiente pregunta dirigida a uno de los padres o acudientes informantes: ¿Durante los últimos 7 días, (nombre del niño) vio televisión o jugó video juegos? Aquellos que respondían de una manera positiva, se 
les indagaba acerca de la frecuencia del evento por medio de la pregunta: ¿Cuántos días (nombre del niño) vio televisión o jugó video juegos? seguida de: ¿En esos días, cuánto tiempo dedicó (nombre del niño) a ver televisión o a jugar con video juegos? En el caso de que el tiempo reportado presentará variaciones entre los días se formulaba la pregunta: ¿En los últimos 7 días, cuánto tiempo en total (nombre del niño) vio televisión o jugó con video juegos?

Dependiendo de las respuestas dadas por los informantes, los niños eran clasificados en dos categorías: menos de 2 horas diarias y 2 horas diarias o más. Este punto de corte ha sido definido por la Academia Americana de Pediatría como el criterio para clasificar aquellos niños que tienen o no un uso excesivo de televisión o video-juegos (20).

Variable independiente

Para el presente análisis se definieron 5 niveles urbanización, teniendo en cuenta las categorías en las que el gobierno colombiano divide los distritos y municipios del país de acuerdo a su población y nivel de ingresos. El nivel 1 correspondió a áreas rurales y a poblaciones menores o iguales a 10000 habitantes, el nivel 2 entre 10001 y 30000 habitantes, el nivel 3 entre 30001 y 1000000 habitantes, el nivel 4, a las áreas urbanas de las ciudades de Barranquilla, Medellín y Cali, con los municipios de Jamundí, Yumbo, Bello e Itagui y el nivel 5 al área urbana de Bogotá con los municipios de Soacha y Chia.

\section{Covariables}

Las siguientes covariables fueron utilizadas en el estudio: subgrupos de edad (5 a 8 años y 9 a 12 años), género y posición socioeconómica de la familia.

La posición socioeconómica fue determinada de acuerdo al SISBEN; un índice desarrollado por el Departamento de Planeación Nacional de Colombia, el cual tiene en cuenta características sociodemográficas, condiciones de vida y acceso a servicios públicos. En las características sociodemográficas se incluyen el estatus de empleo del jefe de hogar, el ingreso familiar y el nivel educativo (21). Las condiciones físicas de la vivienda y la calidad de los materiales de construcción fueron evaluadas en la dimensión de condiciones de vida. En servicios públicos se determinó el acceso a alcantarillado, agua potable, recolección de basuras y electricidad. Teniendo en cuenta los atributos 
mencionados se obtuvieron 6 niveles de posición socioeconómica, siendo 1 el más bajo y 6 el más alto. Debido a que las categorías 4 a 6 comparten algunas similitudes, se decidió colapsarlas en una, con el objeto de incrementar la eficiencia de los análisis.

\section{Análisis estadístico}

Se realizó una descripción de las características de la población de estudio. Con el propósito de determinar las asociaciones entre niveles de urbanización y el haber visto televisión o jugar con video juego por 2 horas o más, se llevó a cabo un análisis de regresión logística (22). En una primera etapa se calcularon los OR de prevalencia crudos entre la variable independiente, las covariables seleccionadas y la variable resultado. Se examinó posteriormente la colinearidad entre las variables por medio de la prueba de factor incremental de la varianza (VIF). Teniendo en cuenta los estudios previos realizados acerca del tema el modelo fue controlado por grupos de edad (5 a 8 años y 9 a 12 años), género y posición socioeconómica de la familia, por ser estas posibles variables de confusión.

El análisis logístico realizado tuvo en cuenta que la información analizada provenía de un muestreo poblacional complejo, en el cual las probabilidades de selección no eran iguales; existía, además, un efecto de conglomeración y varias etapas de selección muestral. Debido a estos aspectos, los análisis fueron realizados utilizando los comandos svy del programa Stata Intercool versión 9 (23).

\section{RESULTADOS}

Características de la población estudiada

En la Tabla 1 se describen las características sociodemográficas de la población estudiada. Se obtuvo respuesta de los tiempos dedicados a ver televisión y jugar con video juegos en 13090 niños en edades comprendidas entre los 5 a 12 años de edad. La distribución por sexo fue aproximadamente igual (50,5\% niñas), la edad media fue de 8,5 años y una proporción ligeramente mayor de niños entre los 9 a 12 años (51 \%). La mayoría de los niños pertenecían a familias con baja posición socioeconómica (42,1 \% con nivel 1 de SISBEN). Con respecto a los niveles de urbanización se encontró que la mayoría de los niños residían en áreas rurales y en municipios con poblaciones menores o 
iguales a 10.000 habitantes (Nivel 1 de urbanización: 71,4 \%). El 56,3 \% de los niños dedicaron 2 o más horas diarias a ver televisión o jugar video juegos.

Tabla 1. Características sociodemográficas en 13090 niños

\begin{tabular}{lcc}
\multicolumn{3}{c}{ entre los 5 a 12 años } \\
\hline \multicolumn{2}{c}{ Caracteristicas } & $n$ \\
\hline Sexo & 6608 & 50,5 \\
Nin̄o & 6482 & 49,5 \\
Niña & & \\
Grupos de edad, años & 6416 & 49,0 \\
5-8 & 6674 & 51,0 \\
9-12 & & \\
Posición socioeconómica de & & \\
acuerdo a puntaje del SISBEN & 5517 & 42,1 \\
Nivel 1 & 4749 & 36,3 \\
Nivel 2 & 2587 & 19,6 \\
Nivel 3 & 257 & 2,0 \\
Niveles 4 a 6 & & 71,4 \\
Niveles de urbanización & 9348 & 6,8 \\
Nivel 1 & 896 & 11,2 \\
Nivel 2 & 1465 & 5,8 \\
Nivel 3 & 754 & 4,8 \\
Nivel 4 & 630 & \\
Nivel 5 & & \\
\hline
\end{tabular}

Factores asociados a haber dedicado 2 o más horas diarias a ver televisión o jugar video-juegos

En la Tabla 2 se pueden observar los resultados de los modelos de regresión logística desagregados por características seleccionadas.

Tabla 2. OR de prevalencia de haber dedicado 2 horas o más diarias a ver televisión o jugar video-juegos en niños de 8 a 12 años

\begin{tabular}{|c|c|c|c|c|c|c|}
\hline Caracteristicas & $\begin{array}{c}\text { OR de } \\
\text { prevalencia } \\
\text { no ajustados }\end{array}$ & $95 \% \mathrm{Cl}$ & $p$ & $\begin{array}{c}\text { *OR de } \\
\text { prevalencia } \\
\text { ajustados }\end{array}$ & $\mathrm{Cl}$ & $p$ \\
\hline \multicolumn{7}{|l|}{ Sexo } \\
\hline Niño & 1 & & & 1 & & \\
\hline Niña & 1,05 & $(0,93-1,18)$ & 0,408 & 1,05 & $(0.93-1.18)$ & 0,426 \\
\hline \multicolumn{7}{|l|}{ Grupos de edad } \\
\hline 5-8 aก้̂os & 1 & & & 1 & & \\
\hline $9-12$ & 1,16 & $(1,01-1,34)$ & 0,029 & 1,19 & $(1,03-1,37)$ & 0,016 \\
\hline \multicolumn{7}{|l|}{ Posición } \\
\hline Nivel 1 & 1 & & & 1 & & \\
\hline Nivel 2 & 1,77 & $(1,45-2,15)$ & 0,000 & 1,54 & $(1,25-1,89)$ & 0,000 \\
\hline Nivel 3 & 2,19 & $(1,71-2,80)$ & 0,000 & 1,79 & $(1,38-2,33)$ & 0,000 \\
\hline Niveles 4 a 6 & 1,91 & $(1,04-3,50)$ & 0,035 & 1,42 & $(0,76-2.64)$ & 0,261 \\
\hline \multicolumn{7}{|l|}{$\begin{array}{l}\text { Nivel de } \\
\text { urbanización }\end{array}$} \\
\hline Nivel 1 & 1 & & & 1 & & \\
\hline Nivel 2 & 0,86 & $(0,73-1,02)$ & 0.097 & 1,33 & $(0,89-1,99)$ & 0,161 \\
\hline Nivel 3 & 1,24 & $(1,07-1,44)$ & 0,004 & 1,35 & $(1,00-1,80)$ & 0,043 \\
\hline Nivel 4 & 1,34 & $(1,09-1,65)$ & 0,005 & 1,61 & $(1,16-2,23)$ & 0,004 \\
\hline Nivel 5 & 1,91 & $(1,48-2,47)$ & 0,000 & 1,70 & $(1,17=2,46)$ & 0,005 \\
\hline
\end{tabular}

Número de observaciones: 13 090; * Modelo ajustado por sexo, grupos de edad, posición socioeconómica y niveles de urbanización 
En el modelo no ajustado, los niños en edades comprendidas entre los 9 a 12 años tuvieron más probabilidad de haber dedicado 2 o más horas diarias a ver televisión o jugar video-juegos con respecto a aquellos en el grupo de edad de 5 a 8 años (OR de prevalencia: 1,16 IC 95 \% 1,01-1,34). La dirección de esta asociación fue similar en el modelo ajustado (OR de prevalencia: 1,19 IC $95 \%$ 1,03-1,37).

Con respecto a la posición socioeconómica, aquellos niños en hogares con niveles de SISBEN diferente a 1 tuvieron más probabilidades de presentar una exposición excesiva televisión o video-juegos, con un OR de prevalencia mayor en el nivel 3 (2,19 IC 95 \% 1,71-2,80). En el modelo ajustado la asociación persistió en los niveles 2 y 3 de SISBEN.

Se puede observar finalmente, que una vez realizado el ajuste por edad, sexo y posición socioeconómica, la probabilidad de ver televisión o jugar con video-juegos por 2 horas o más es cada vez mayor, a medida que el nivel de urbanización se incrementaba, siendo esta asociación estadísticamente significativa a partir del nivel 3 de urbanización (Tabla 2 y Figura 1).

Figura 1. OR de prevalencia ajustados de haber dedicado 2 horas o más diarias a ver televisión o jugar video-juegos de acuerdo a niveles de urbanización, en niños de 6 a 12 años

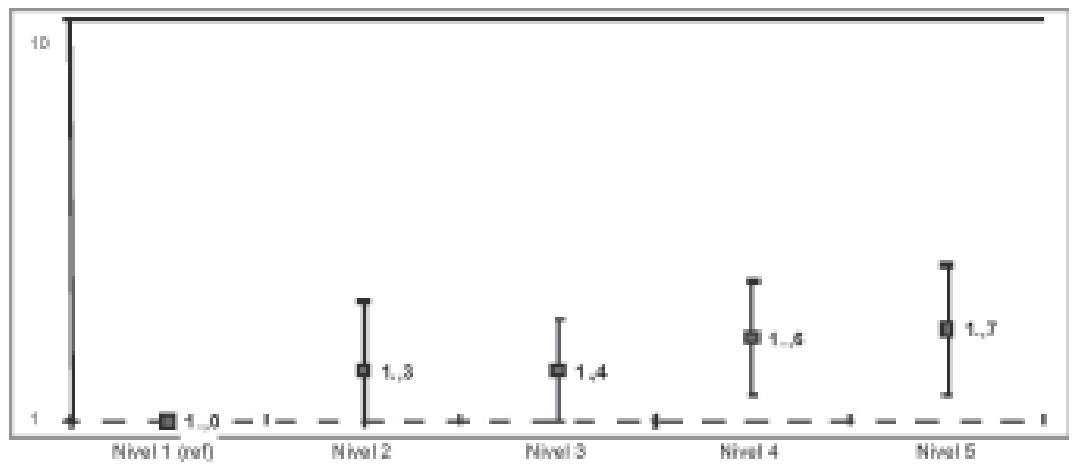

DISCUSIÓN

Este estudio encontró una asociación positiva y gradual, entre los niveles de urbanización y el haber dedicado 2 o más horas diarias a ver televisión o usar video-juegos en una muestra representativa de niños colombianos de 5 a 12 años. Los resultados persistieron después de realizar ajustes por potenciales variables que generan confusión. 
Este es el primer estudio en América Latina que explora los vínculos entre nivel de urbanización y uso de medios electrónicos de entretenimiento y complementa un estudio previo en el que demostramos una asociación significativa entre obesidad infantil y uso excesivo de televisión y video-juegos en esta misma población (24) El adecuado tamaño de muestra, representativo de la población infantil colombiana y la información proveniente de diversas áreas de Colombia, permitieron obtener un perfil amplio del fenómeno.

Diversos aspectos podrían explicar los resultados de este estudio. Las relaciones entre niveles urbanización y el uso de entretenimientos electrónicos pueden estar mediadas por los crecientes procesos de globalización, que son más evidentes en grandes asentamientos urbanos (25).

Es posible además, que los patrones de vida urbana propicien una mayor utilización de medios electrónicos de entretenimiento. Aspectos como las condiciones de seguridad objetiva y percibida y las dinámicas familiares pueden jugar un papel destacado (26-28).

Algunos estudios han tratado de establecer las asociaciones entre las percepciones de seguridad del vecindario y el tiempo en que los niños y adolescentes dedican a ver televisión (26). No obstante, los hallazgos no han sido consistentes, debido en parte a que el interés se ha centrado en valorar los niveles de criminalidad, sin considerar otras dimensiones estrechamente vinculadas a la vida urbana, tales como la percepción y medición objetiva de los niveles de tráfico automotor, los cuales pueden influir en la decisión de padres o cuidadores para que los niños permanezcan más tiempo en casa (27). Este aspecto puede estar relacionando además, con el interés de estos de reducir la exposición de los menores a la contaminación ambiental, la cual se ha establecido como alta en áreas urbanas (27-29).

Producto de las transformaciones sociales que el país ha experimentado desde el siglo pasado, se ha aumentado la participación de ambos padres en actividades laborales fuera del hogar. Es posible que este fenómeno pudiera conllevar a una menor dedicación a actividades del hogar por parte de padres y madres residentes en áreas urbanas, reduciendo el tiempo que dedican a supervisar las actividades de los niños cuando están en casa e incrementando, a su vez, un mayor consumo de televisión y video juegos (30-31). En este contexto, la televisión y los entretenimientos electrónicos comienzan a suplir algunas funciones tradicionalmente ejercidas por la familia u otras instituciones sociales (32). 
Con el creciente incremento en países no desarrollados de los problemas de salud vinculados con el uso de televisión y video-juegos, como la obesidad infantil y sus efectos negativos en la edad adulta, el estudio del tema cobra relevancia para la salud pública.

Son diversas las limitaciones metodológicas de este estudio, el cual por su corte transversal y carácter exploratorio, no permite establecer un vinculo causal entre las variables estudiadas. Adicionalmente, la información del tiempo dedicado a ver televisión o jugar video-juegos, la principal variable de exposición en este estudio, fue suministrada por los padres o acudientes del niño, con la posible subestimación o sobrestimación de la información suministrada. Sin embargo, la mayoría de investigaciones realizados hasta el momento en donde el uso de televisión y video-juegos en niños de esta edad han utilizado instrumentos similares como cuestionarios o reportes de padres. Futuros estudios deberán abordar la validez de estos instrumentos o desarrollar métodos de medición directa de estos hábitos, dado su impacto en salud pública.

A pesar de estas limitaciones, los resultados de este estudio indican que los niños que viven en zonas con moderados y altos niveles de urbanización deben ser considerados como grupos en riesgo de uso excesivo de televisión y videojuegos. Los programas de prevención de obesidad infantil y promoción de actividad física deben incluir estrategias encaminadas a limitar el uso de entretenimiento electrónico especialmente en centros urbanos de gran y mediano tamaño. Adicionalmente, se destaca la importancia de monitorear este comportamiento en las futuras encuestas de nutrición en Colombia *

Agradecimientos. Los autores desean agradecer el apoyo recibido por el Instituto Colombiano de Bienestar Familiar y PROFAMILIA. Catalina Borda y Ana Maria Peñuela brindaron aportes en la concepción de este manuscrito.

\section{REFERENCIAS}

1. Story M, French S. Food advertising and marketing directed at children and adolescents in the US. Int J Behav Nutr Phys Act 2004; 10:1-3.

2. Bercedo A, Redondo C, Capa L, González MA. Hábito televisivo en los niños de Cantabria. An Esp Pediatr 2001; 54: 44-52.

3. McNeal J. Los niños como consumidores de productos sociales y comerciales. Wasghinton D.C.: Organización Panamericana de la Salud; 2000.

4. Children's Television Workshop, New York, NY [Internet] Disponible en: http://www.eric.ed.gov/ ERICDocs/data/ericdocs2sql/content_storage_01/0000019b/80/23/77/81.pdf . Consultado Enero de 2008. 
5. Dietz WH, Gortmaker SL. Do we fatten our children at the television set? Obesity and television viewing in children and adolescents. Pediatrics 1985;75:807-12.

6. Crespo CJ, Smit E, Troiano RP, Bartlett SJ, Macera CA, Andersen RE. Television watching, energy intake, and obesity in US children: results from the third -National Health and Nutrition Examination Survey, 1988-1994. Arch Pediatr Adolesc Med. 2001;155:360365.

7. Marshall SJ, Biddle SJ, Gorely T, Cameron N, Murdey I. Relationships between media use, body fatness and physical activity in children and youth: a meta-analysis. Int J Obes Relat Metab Disord 2004; 28: 1238-1246.

8. Hernández B, Gortmaker SL, Colditz GA, Peterson KE, Laird NM, Parra-Cabrera S. Association of obesity with physical activity, television programs and other forms of video viewing among children in Mexico city. Int J Obes Relat Metab Disord. 1999;23:845-54.

9. Lobelo F, Dowda M, Pffeifer KA, Pate RR. Electronic Media Exposure and Its Association With Activity-Related Outcomes in Female Adolescents: Cross-Sectional and Longitudinal Analyses. J Phys Act Health In press

10. Dibb S, Harris L. A spoonful of sugar. Television food advertising aimed at children: An international comparative study. London: Consumers International; 1996.

11. Gortmaker SL, Must A, Sobol AM, Peterson K, Colditz GA, Dietz WH. Television viewing as a cause of increasing obesity among children in the United States 1986-1990. Arch Pediatr Adolesc Med.1996;150:356-62

12. Dietz WH, Strasburger VC. Children, adolescents and television. Curr Probl Pediatr 1991;21:831.

13. Gentile DA, Walsh DA, Ellison PR, Fox M, Cameron J. 2004. "Media violence as a risk factor for children: A longitudinal study" [Dissertation]. Chicago: American Psychological Society 16th Annual Convention; 2004.

14. Dodge KA, Bates JF, Petitt CS. Mechanisms in the cycle of violence. Science. 1990;250:167883.

15. Singer MI, Miller DB, Guo S, Flannery DJ, Frierson T, Slovak K. Contributors to violent behavior among elementary and middle school children. Pediatrics. 1999;104:878-84.

16. Paik $\mathrm{H}$, Comstock $\mathrm{G}$. The effects of television violence on antisocial behavior: a meta-analysis. Commun Res. 1994;21:516:46.

17. Wood W, Wong FY, Chachere JG. Effects of media violence on television on viewers aggression in unconstrained social interaction. Psychol Bull. 1999;109:371-83.

18. Comisión Nacional de Televisión. [Internet] Disponible en: http://www.cntv.org.co/ . Consultado septiembre de 2006.

18. Instituto Colombiano de Bienestar Familiar. Encuesta Nacional de la Situación Nutricional en Colombia. Instituto Colombiano de Bienestar Familiar :Bogotá D.C.; 2005.

20. American Academy of Pediatrics, Committee on Public Education. Children, Adolescents and Television. Pediatrics 2001;107 (2), 423-426.

21. Castaño E. Metodología para la determinación de los puntos de corte del índice SISBEN para la caracterización de la población pobre. Bogotá: Misión Social, DNP; 1995.

22. Kleinbaum D, Kupper L, Muller K. Applied regression analysis and other multivariate methods. 2nd ed. Boston: PWS-Kent; 1987.

23. Statacorp, Stata Statistical Software: release 9. Stata Corporation: College Station, TX;2005.

24. Gomez LF, Parra DC, Lobelo F, Samper B, Moreno J, Jacoby E, Lucumí DI, Matsudo S, Borda C. Television viewing and its association with overweight and obesity in Colombian children: results from the National Nutrition Survey (ENSIN 2005): A cross sectional study. Int J Behav Nutr Phys Act (IJBNPA). 2007;4:41

25. Crenshaw EM, Robison KK. Globalization and the Digital Divide: The Roles of Structural Conduciveness and Global Connection in Internet Diffusion. Social Science Quarterly 2006; 87:190-207.

26. Burdette $H$, Whitaker R. A National study of neighboorhods safety, outdoor play, television viewing, and obesity in preschool children. Pediatrics 2005;116:657-662. 
27. Frenz P, Vega J, Marchetti N, Torres J, Kopplin E, Delgado I, Vega F. Chronic exposure to environmental lead in Chilean infants. Rev Med Chil. 1997;125:1137-44.

28. Venn A, Lewis S, Cooper M, Hubbard R, Hill I, Boddy R, et al. Local road traffic activity and the prevalence, severity, and persistence of wheeze in school children: combined cross sectional and longitudinal study. Occup Environ Med. 2000;57: 152-8.

29. Lopez R, Hynes P. Obesity, Physical Activity, and the urban environment: public health research needs. Environmental Health: A global Access. Science Source; 2006. 5:25. doi: 10.1186/ 1476-069X-5-25.

30. Departamento Administrativo Nacional de Estadísticas. [Internet] Disponible en: http:// www.dane.gov.co/files/investigaciones/empleo/. Consultado Septiembre 2006.

31. Salmon J, Timperio A, Teldford A, Carver A, Crawford D. Association of family environment with children's television Beijing and with low level of physical activity. Obesity research 2005 13(11):1940-1951.

32. Silverblatt A. Media as Social Institution. American Behavioral Scientist 2004;48:35-41. 